\title{
COVID-19 in patients with HIV-1 infection: a single-centre experience in northern Italy
}

\author{
Leonardo Calza ${ }^{1}$ (D) Isabella Bon ${ }^{2} \cdot$ Marina Tadolini $^{1} \cdot$ Marco Borderi $^{1} \cdot$ Vincenzo Colangeli $^{1} \cdot$ Lorenzo Badia $^{1}$. \\ Gabriella Verucchi $^{1} \cdot$ Giada Rossini $^{2} \cdot$ Caterina Vocale $^{2} \cdot$ Paolo Gaibani $^{2} \cdot$ Pierluigi Viale $^{1} \cdot$ Luciano Attard $^{1}$
}

Received: 18 June 2020 / Accepted: 23 July 2020 / Published online: 3 August 2020

(c) The Author(s) 2020

\begin{abstract}
Background Since the end of February 2020, the Coronavirus Disease 2019 (COVID-19) outbreak rapidly spread throughout Italy and other European countries, but limited information has been available about its characteristics in HIV-infected patients.

Methods We have described a case series of patients with HIV infection and COVID-19 diagnosed at the S.Orsola Hospital (Bologna, Italy) during March and April, 2020.

Results We reported a case series of 26 HIV-infected patients with COVID-19. Nineteen subjects were men, the median age was 54 years, $73 \%$ of patients had one or more comorbidities. Only 5 patients with interstitial pneumonia were hospitalized, but there were no admissions to intensive care unit and no deaths.

Conclusions In our experience, COVID-19 associated with HIV infection had a clinical presentation comparable to the general population and was frequently associated with chronic comorbidities.
\end{abstract}

Keywords Coronavirus $\cdot$ HIV $\cdot$ Comorbidity $\cdot$ Pneumonia $\cdot$ Protease inhibitors

\section{Introduction}

In early December 2019, an interstitial pneumonia of unknown origin emerged in Wuhan city, the capital of Hubei province in China. The pathogen has been identified as a novel betacoronavirus, which has subsequently been named Severe Acute Respiratory Syndrome Coronavirus 2 (SARSCoV-2), and the illness caused by SARS-CoV-2 has been defined Coronavirus disease 2019 (COVID-19) [1,2].

During the first 2 months of the outbreak, COVID-19 spread rapidly throughout China and caused varying degrees of illness. In a retrospective analysis of 1099 patients with laboratory-confirmed COVID-19 from 30 provinces in

Leonardo Calza

leonardo.calza@unibo.it

1 Department of Medical and Surgical Sciences, Unit of Infectious Diseases, "Alma Mater Studiorum" University of Bologna, S. Orsola-Malpighi Hospital, via G. Massarenti 11 - I-40138, Bologna, Italy

2 Department of Experimental, Diagnostic and Specialty Medicine, Unit of Microbiology, "Alma Mater Studiorum" University of Bologna, S. Orsola Hospital, Bologna, Italy
China, the median incubation period was 4 days, the most common symptoms were fever and cough, and the most frequent laboratory abnormalities were lymphocytopenia, increased lactate dehydrogenase, and increased C-reactive protein. On chest computed tomography, ground-glass opacity was the most common radiologic finding, $5 \%$ of patients were admitted to an intensive care unit (ICU), and $1.4 \%$ died $[1,2]$. In a meta-analysis of 43 studies involving 3600 patients, bilateral pneumonia was present in $73.2 \%$ of cases, the estimated proportion of severe cases was $25.6 \%$, and the case-fatality rate was $3.6 \%$ [3].

In the following weeks, the COVID-19 outbreak became a global pandemic and on February 20, 2020, the first patient with SARS-CoV-2 pneumonia was diagnosed in Lombardy region, in northern Italy. Since then, the increasing number of cases reported throughout the country led Italy to be the second most affected country in the world, after the United States, as of the end of March 2020 [4].

A matter of debate includes frequency and seriousness of COVID-19 in immune compromised patients, such as those with human immune deficiency virus-1 (HIV-1) infection. To date, clinical data about COVID-19 in HIV-1-infected 
patients are lacking and descend only from a few case series [5-11].

\section{Case series}

Between March 1, 2020 and April 30, 2020, a total of 756 SARS-CoV-2 infections were diagnosed at the S.Orsola Hospital in Bologna (Emilia-Romagna region, Italy). During these 2 months, 26 cases of SARS-CoV-2 infections were diagnosed in patients with HIV-1 infection. Therefore, the overall prevalence of coinfection of SARS-CoV-2 and HIV-1 among all cases of COVID-19 diagnosed in this period was $3.4 \%$ [interquartile range (IQR), 2.1-4.8]. The incidence of SARS-Cov-2 infection among HIV-positive persons referring to our hospital as of 30 April 2020 was $0.94 \%$ (IQR, $0.67-1.19)$.

Characteristics, treatments and outcomes of 26 HIVinfected patients with COVID-19 are summarized in Table 1. All patients gave informed consent. Overall, 19 (73\%) were men, median age was 53.8 years (range, 28-80), all were currently treated with combination antiretroviral therapy (cART), and 22 subjects (85\%) had a plasma HIV RNA below 50 copies $/ \mathrm{mL}$. Reported CD4 + T lymphocyte count and HIV RNA value were the last measurements before the COVID-19 diagnosis. Current CD4 + lymphocyte count was above 350 cells $/ \mathrm{mm}^{3}$ in 22 individuals (85\%), 5 (19\%) had a previous diagnosis of an AIDS-defining condition, and current cART included one boosted protease inhibitor (PI) in 6 cases (23\%). Nineteen patients (73\%) had one or more comorbidities.

Diagnosis of COVID-19 was made by detection of SARS-CoV-2 RNA in oro- and/or naso-pharyngeal swab specimens by real-time RT-PCR targeting regions in the $N$ gene, following the US CDC protocol. Clinical diagnosis was represented by upper respiratory tract infection in 20 cases (77\%) and interstitial pneumonia in $6(23 \%)$. At diagnosis, the median duration of symptoms was 4.2 days, and most frequent symptoms were fever $>38^{\circ} \mathrm{C}$, cough, fatigue, myalgia, and tachypnea. A reduction in $\mathrm{O}_{2}$ saturation $<95 \%$ in ambient air was present in 7 subjects (27\%), but only 2 patients (8\%) had an initial respiratory failure with a $\mathrm{PaO}_{2} /$ $\mathrm{FiO}_{2}$ ratio $<300$ at arterial blood gas analysis.

Only 5 patients (19\%) with interstitial pneumonia were hospitalized, while other 21 subjects $(81 \%)$ spent their disease period at home. The five hospitalized patients had a median age of 55.1 years (IQR, 46.2-68.3) and a median CD4 + lymphocyte count of 622 cells $/ \mathrm{mm}^{3}$ (IQR, 458-814). All hospitalized patients had current CD4 + lymphocyte count above 350 cells $/ \mathrm{mm}^{3}$. At diagnosis, 6 patients (23\%) were receiving a PI-based cART, including darunavir-cobicistat in 5 cases and darunavir-ritonavir in one case. Sixteen patients $(61.5 \%)$ were receiving a cART including tenofovir disoproxil fumarate or tenofovir alafenamide. A transitional change in cART was made in other 6 patients who were treated with a non-boosted PI-based regimen, because of the potential activity of HIV PIs against the Coronavirus protease. So, darunavir/cobicistat replaced rilpivirine in 5 cases and efavirenz in one case. With regard to other drug treatments, we prescribed hydroxychloroquine in 13 subjects $(50 \%)$ and enoxaparin in $6(23 \%)$, while tocilizumab and corticosteroids were not employed.

A clinical recovery was obtained in 22 patients ( $85 \%$ ) and a clinical improvement in the remaining 4 (15\%), while there were no admissions to ICU and no deaths. The five hospitalized patients were discharged after a median of 9.2 days, and the median global duration of symptoms before recovery in all observed patients was 7.5 days.

\section{Discussion}

Clinical course and outcome of COVID-19 among patients with HIV-1 infection are still unknown. Some authors have speculated that HIV-1-infected patients on cART may have a lower risk for COVID-19 and related complications because of the in vitro activity of some antiretroviral drugs against SARS-CoV-2 and their defective cellular immunity, leading to a decreased possibility of cytokine dysregulation and consequent severe lung damage [12, 13]. On the contrary, other authors have suggested an increased risk for COVID-19 due to HIV-1-related immunosuppression [14].

In some recent cohort studies, HIV-infected persons hospitalized for COVID-19 had similar clinical characteristics and outcomes with other hospitalized cohorts of HIV-uninfected patients, and risk of COVID-19 and severe disease in suppressed HIV-positive people seems to be comparable with the general population [5-11].

In our experience, HIV-infected patients accounted for $3.4 \%$ of all patients with diagnosis of SARS-CoV-2 infection made at our hospital during the observation period. This prevalence is higher than that reported by Blanco JL et al. at the Hospital Clinic of Barcelona, Spain (0.92\%) [7], but these authors described only COVID-19 patients co-infected with HIV-1 among consecutive subjects admitted to their hospital, and not co-infected patients who were not hospitalized. Moreover, not all HIV-positive patients of our clinics were tested for SARS-CoV-2, and probably there were many cases of asymptomatic SARS-CoV-2 infections among HIVpositive people, likewise in the general population, so these data should be considered with great caution.

In an observational prospective study performed at the Hospital Cajal of Madrid, Spain, Vizcarra P et al. [8] have described 51 HIV-infected patients with COVID-19 and compared their characteristics with 1,288 HIV-positive 
Table 1 Demographic data, clinical characteristics, treatment, and outcomes of patients with COVID-19 and HIV infection
Patients, $n$

26

Demographics, HIV baseline status and comorbidites

Men, $n(\%)$

$19(73.1)$

Caucasian, $n(\%)$

$25(96.1)$

Age (years), median (IQR)

$53.8(42.5-64.7)$

HIV transmission risk category, $n(\%)$

IDU

$4(15.4)$

MSM

$14(53.8)$

Heterosexual

$8(30.8)$

Current CD4 + lymphocyte count (cells $\left./ \mathrm{mm}^{3}\right)$, median (IQR)

Patients with CD4 + lymphocyte count $<350$ cells $/ \mathrm{mm}^{3}, n(\%)$

Patients with HIV RNA $<50$ copies/mL, $n(\%)$

Patients with AIDS diagnosis, $n(\%)$

Duration of HIV infection (years), median (IQR)

Current ART

NNRTIs, $n(\%)$

Boosted PIs, $n(\%)$

$566(304-821)$

4 (15.4)

$22(84.6)$

5 (19.2)

$16.2(8.4-25.3)$

INSTIs, $n(\%)$

Patients with one or more comorbidities, $n(\%)$

Patients with arterial hypertension, $n(\%)$

Patients with diabetes mellitus, $n(\%)$

Patients with BMI $>30 \mathrm{~kg} / \mathrm{m}^{2}, n(\%)$

Patients with asthma or chronic respiratory diseases, $n(\%)$

Patients with hypothyroidism, $n$ (\%)

Clinical presentation at diagnosis

Diagnosis

Upper respiratory tract infection, n. (\%)

Interstitial pneumonia, n. (\%)

Interstitial pneumonia with ARDS, n. (\%)

Duration of symptoms before diagnosis (days), median (IQR)

Temperature $\geq 38^{\circ} \mathrm{C}, n(\%)$

Cough, $n(\%)$

Myalgia, $n(\%)$

Fatigue, $n(\%)$

Dyspnoea, $n(\%)$

Respiratory rate $>20$ (breaths per min), $n(\%)$

$\mathrm{O}_{2}$ saturation in ambient air $<95 \%, n(\%)$

$\mathrm{PaO}_{2} / \mathrm{FiO}_{2}$ ratio $<300, n(\%)$

White blood cell count (cells per $10^{6} / \mathrm{L}$ ), median (IQR)

Lymphocyte count (cells per $10^{6} / \mathrm{L}$ ), median (IQR)

Patients with lymphocyte count $<1000$ cells per $10^{6} / \mathrm{L}, n(\%)$

Patients with platelet count $<150,000$ cells per $10^{6} / \mathrm{L}, n(\%)$

LDH (U/L), median (IQR)

C-reactive protein $(\mathrm{mg} / \mathrm{dL})$, median (IQR)

$13(50)$

$6(23.1)$

$11(42.3)$

$19(73.1)$

$11(42.3)$

4 (15.4)

$4(15.4)$

3 (11.6)

3 (11.6)

$20(76.9)$

$6(23.1)$

0

$4.2(2.1-6.5)$

$19(73.1)$

$14(53.8)$

$11(42.3)$

$11(42.3)$

3 (11.6)

4 (15.4)

7 (26.9)

2 (7.7)

$7.61(3178-12,339)$

1555 (780-2331)

$12(46.1)$

$10(38.5)$

618 (309-1108)

$4.2(0.71-9.2)$

Treatment and outcomes

Hospitalization, $n(\%)$

5 (19.2)

Admission to an ICU, $n(\%)$

Non-invasive mechanical ventilation, $n(\%)$

0

Darunavir/ritonavir or darunavir/cobicistat, $n(\%)$

$12(46.1)$

Hydroxychloroquine, $n(\%)$

$13(50)$

Azithromycin, $n(\%)$

$6(23.1)$

Enoxaparin, $n(\%)$ 
Table 1 (continued)

\begin{tabular}{ll}
\hline Tocilizumab, $n(\%)$ & 0 \\
Corticosteroids, $n(\%)$ & 0 \\
Recovery, $n(\%)$ & $22(84.6)$ \\
Clinical improvement, $n(\%)$ & $4(15.4)$ \\
Death, $n(\%)$ & 0 \\
Duration of hospitalization (days), median (IQR) & $9.2(6.5-12.3)$ \\
Total duration of symptoms (days), median (IQR) & $7.5(4.9-10.2)$ \\
\hline
\end{tabular}

$I Q R$ interquartile range, $I D U$ injection drug users, MSM men who have sex with men, DRMs drug resistance mutations, NNRTIs non-nucleoside reverse transcriptase inhibitors, PIs protease inhibitors, INSTIs integrase strand transfer inhibitors, $B M I$ body mass index, $A R D S$ acute respiratory distress syndrome, $L D H$ lactic dehydrogenase, $I C U$ intensive care unit

subjects without COVID-19. In this study, clinical course, radiological findings, and outcome of SARS-CoV-2 infection in HIV-positive persons were similar to those reported in the general population, and HIV-infected patients with COVID-19 had a higher prevalence of comorbidities (mostly hypertension and diabetes) than HIV-infected subjects without COVID-19. The incidence of COVID-19 among HIVinfected people reported by Vizcarra $\mathrm{P}$ et al. was $1.2 \%$, or rather slightly higher in comparison with our cohort.

In a German series of $33 \mathrm{HIV}$-infected patients with COVID-19, $91 \%$ of subjects recovered and $76 \%$ had a mild form of disease, so morbidity and mortality of COVID19 were comparable to those reported in other cohorts of HIV-negative patients [9].

In a case series of $31 \mathrm{HIV}$-infected patients hospitalized for COVID-19 in a large tertiary care centre in New York City [10], clinical features and outcomes were similar to those described in other hospitalized cohorts. The overall prevalence of COVID-19 among HIV-infected people (approximately 1.4\%) was slightly lower than that reported in our cohort. In this study, all patients were on stable cART with undetectable HIV viral load, and most subjects had CD $4+\mathrm{T}$ cell count above 200 cells $/ \mathrm{mm}^{3}$, as much as observed in another case series of $27 \mathrm{HIV}$-positive persons with COVID-19 in New Jersey [11]. In another report of 88 HIV-infected patients with COVID-19 hospitalized in New York City, there were no differences in adverse outcomes in comparison with a demographically similar patient group. Moreover, HIV-positive patients had greater proportions of smoking and comorbidities than similar HIV-negative comparators [15]. Therefore, in these cohorts, SARS-CoV-2 infections do not seem to act as an opportunistic pathogen in the setting of HIV disease.

In our study, the median age of patients was 54 years and 23 subjects $(88 \%)$ were younger than 70 years, but they had a significant prevalence of chronic comorbidities (73\%), represented mostly by hypertension, diabetes and obesity, such as for HIV-1-negative COVID-19 patients [1-3]. Our patients showed in most cases a good immunological condition, so a low CD4 + lymphocyte count did not seem to be associated with an increased risk of COVID-19, while comorbidities were often present. A current PI-based cART was present in only 6 persons, and in other 6 cases, the antiretroviral regimen was adapted to a PI-based combination, even though clinical data about efficacy of HIV-1 PIs in patients with COVID-19 are still inconclusive.

In a few case reports, lopinavir/ritonavir associated with adjuvant drugs for interstitial pneumonia in COVID-19 patients had a more evident therapeutic effect than adjuvant drugs alone in improving clinical symptoms and reducing the SARS-CoV-2 viral load [16, 17]. However, in a randomized, controlled trial involving 199 Chinese patients with COVID-19 and treated with lopinavir/ritonavir for 14 days in addition to standard care or standard care alone, no significant benefit was observed in the lopinavir/ritonavir group. In detail, the PI-based treatment was not associated with a difference from standard care in the time to clinical improvement, mortality, and duration of detectable SARS-CoV-2 viral load [18]. No clinical data are currently available about other HIV PIs as treatment of COVID-19. A case series provided preliminary evidence that darunavir at the usual dosage did not prevent SARS-CoV-2 infection in HIV-1-positive persons [19].

In conclusion, from our experience, SARS-CoV-2 infection, which had among HIV-1-infected patients a clinical presentation comparable or milder than general population, was not more frequent or more serious among subjects with low CD4 + lymphocyte count, and was frequently associated with chronic comorbidities. Further, enlarged cohort studies are needed to better understand risk and clinical course of COVID-19 among HIV-infected people.

Acknowledgements Open access funding provided by Alma Mater Studiorum - Università di Bologna within the CRUI-CARE Agreement.

Funding This work was supported only by internal funding of the Unit of Infectious Diseases, S. Orsola Hospital, University of Bologna (Italy). 


\section{Compliance with ethical standards}

Conflict of interest All authors declare that they have no conflict of interest.

Open Access This article is licensed under a Creative Commons Attribution 4.0 International License, which permits use, sharing, adaptation, distribution and reproduction in any medium or format, as long as you give appropriate credit to the original author(s) and the source, provide a link to the Creative Commons licence, and indicate if changes were made. The images or other third party material in this article are included in the article's Creative Commons licence, unless indicated otherwise in a credit line to the material. If material is not included in the article's Creative Commons licence and your intended use is not permitted by statutory regulation or exceeds the permitted use, you will need to obtain permission directly from the copyright holder. To view a copy of this licence, visit http://creativecommons.org/licenses/by/4.0/.

\section{References}

1. Huang C, Wang Y, Li X, Ren L, Zhao J, Hu Y, et al. Clinical features of patients infected with 2019 novel coronavirus in Wuhan, China. Lancet. 2020;395:497-506. https://doi.org/10.1016/S0140 -6736(20)30183-5.

2. Zhu N, Zhang D, Wang W, Li X, Yang B, Song J, et al. A novel coronavirus from patients with pneumonia in China, 2019. N Engl J Med. 2020;382:727-33. https://doi.org/10.1056/nejmoa2001 017.

3. Fu L, Wang B, Yuan T, Chen X, Ao Y, Fitzpatrick T, et al. Clinical characteristics of coronavirus disease 2019 (COVID-19) in China: a systematic review and meta-analysis. J Infect. 2020;80:656-65. https://doi.org/10.1016/j.jinf.2020.03.041.

4. Grasselli G, Zangrillo A, Zanella A, Antonelli M, Cabrini L, Castelli A, et al. Baseline characteristics and outcomes of 1591 patients infected with SARS-CoV-2 admitted to ICUs of the Lombardy Region, Italy. JAMA. 2020;323:1574-81. https://doi. org/10.1001/jama.2020.5394.

5. Joob B, Wiwanitkit V. SARS-CoV-2 and HIV. J Med Virol. 2020. https://doi.org/10.1002/jmv.25782.

6. Zhu F, Cao Y, Xu S, Zhou M. Co-infection of SARS-CoV-2 and HIV in a patient in Wuhan city, China. J Med Virol. 2020;92:52930. https://doi.org/10.1002/jmv.25732.

7. Blanco JL, Ambrosioni J, Garcia F, Martinez E, Soriano A, Mallolas J, et al. COVID-19 in patients with HIV: clinical case series. Lancet HIV. 2020;7:e314-6. https://doi.org/10.1016/s2352 $-3018(20) 30111-9$.
8. Vizcarra P, Perez-Elias MJ, Quereda C, Moreno A, Vivancos MJ, Dronda F, et al. Description of COVID-19 in HIV-infected individuals: a single-centre, prospective cohort. Lancet. 2020. https ://doi.org/10.1016/s2352-3018(20)30164-8.

9. Harter G, Spinner CD, Roider J, Bickel M, Krznaric I, Grunwald S, et al. COVID-19 in people living with human immunodeficiency virus: a case series of 33 patients. Infection. 2020. https:// doi.org/10.1007/s15010-020-01438-z.

10. Shalev N, Scherer M, LaSota ED, Antoniou P, Yin MT, Zucker J, et al. Clinical characteristics and outcomes in people living with HIV hospitalized for COVID-19. Clin Infect Dis. 2020. https:// doi.org/10.1093/cid/ciaa635.

11. Okoh AK, Bishburg E, Grinberg S, Nagarakanti S. COVID-19 pneumonia in patients with HIV-a case series. J Acquir Immune Defic Syndr. 2020. https://doi.org/10.1097/qai.000000000000241 1.

12. Dauby N. Potential impact of COVID-19 in people living with HIV: experience from previous 21 st century coronaviruses epidemics. AIDS. 2020;34:1255-6. https://doi.org/10.1097/ qad.0000000000002555.

13. Toombs JM, Van den Abbeele K, Democratis J, Merricks R, Mandal AKJ, Missouris CG, et al. COVID-19 in 3 people living with HIV in the United Kingdom. J Med Virol. 2020. https://doi. org/10.1002/jmv.26178.

14. Mascolo S, Romanelli A, Carleo MA, Esposito V. Could HIV infection alter the clinical course of SARS-CoV-2 infection? When less is better. J Med Virol. 2020. https://doi.org/10.1002/ jmv. 25881.

15. Sigel K, Swartz T, Golden E, Paranjpe I, Somani S, Richter F, et al. COVID-19 and people with HIV infection: outcomes for hospitalized patients in New York City. Clin Infect. 2020. https:// doi.org/10.1093/cid/ciaa880.

16. Ye XT, Luo YL, Xia SC, Sun QF, Ding JH, Zhou Y, et al. Clinical efficacy of lopinavir/ritonavir in the treatment of Coronavirus disease 2019. Eur Rev Med Pharmacol Sci. 2020;24:3390-6. https ://doi.org/10.26355/eurrev_202003_20706.

17. Liu F, Xu A, Zhang Y, Xuan W, Yan T, Pan K, et al. Patients of COVID-19 may benefit from sustained lopinavir-combined regimen and the increase of eosinophil may predict the outcome of COVID-19 progression. Int J Infect Dis. 2020;95:183-91. https ://doi.org/10.1016/j.ijid.2020.03.013.

18. Cao B, Wang Y, When D, Liu W, Wang J, Fan G, et al. A trial of lopinavir-ritonavir in adults hospitalized with severe COVID-19. N Engl J Med. 2020;382:1787-99. https://doi.org/10.1056/nejmo a2001282.

19. Riva A, Conti F, Bernacchia D, Pezzati L, Sollima S, Merli $\mathrm{S}$, et al. Darunavir does not prevent SARS-CoV-2 infection in HIV patients. Pharmacol Res. 2020. https://doi.org/10.1016/j. phrs.2020.104826. 